\title{
Symbiotic Approach in a Modular Mobile Robot
}

\author{
Arvin H. Fernando ${ }^{1}$, Dr. Laurence A. Gan Lim ${ }^{1}$ \\ ${ }^{1}$ Mechanical Engineering Department \\ ${ }^{1}$ De La Salle University \\ ${ }^{1} 2401$ Taft Ave, Manila, 1004 Philippines \\ arvin.fernando@dlsu.edu.ph
}

\begin{abstract}
This paper presents the application of a symbiotic approach in a modular mobile robot. This characteristic behavior might help address the challenges in modular reconfigurable robotoperation. The general model symbiosis algorithm will help decide if the modular part is harmful or beneficial to the performance or task of the robotic system thru the carrying capacity. The symbiotic behavior is presented and implemented in this paper viamodel-baseddesign with the aid of MATLAB Simulink using a 6 wheeled mobile robot with 3 modular body to identify the carrying capacity of the system.Carrying capacity is translated and used as the distance and velocity capacity of the design model robotic system. Carrying capacity is greatly influence by the number species or in our case modules it is shown in this paper that carrying capacity are not fixed in quantities but should be consider as functions of the population sizes and function. The mathematical formulation of the idea is to investigate its consequence. Aside from the population size role or interaction.
\end{abstract}

Key words: Carrying Capacity, Modular Robot, Reconfigurable Robot, Symbiosis.

\section{INTRODUCTION}

Robots are designed to carry out discrete and define tasks and have fixed configuration. Modular and reconfigurable robot shows the promise of great versatility, robustness, flexibility, and low cost. The domain of modular robotic system, self-configuration, self-diagnose, and self-repair are known to be a challenging task[1]. In the field in of mobile robotics there is a demand for a fault detection and diagnose of sensor,actuator, and system component to assure system reliability and safety[2].In a collective robotic system, the probability rises exponentially with increasing system scale and suffer from unanticipated faults.[1] The mechanism and processing of a modular robot is simple and limited which is challenging.
Modular reconfigurable robots (MRR) are composed of modules that can arrange themselves to different configuration to perform varioustask. Modular selfreconfigurable system, size, robustness, and performance have been continuously improving however there are challenges for these systems to realize their promise of adaptability, robustness, and affordability. MRR faces hardware design and Software control challenges[3][4][5] . Modular Robots consist of several independent modules that have different capabilities that can connect or form into a different configuration. These hardwaremechanical structuressuchactuators, sensing communication device, structural strength, connection mechanism, and others are required for it in order to function properly. To perform a taskModular system, have complex software requirements that helps them run independently, as a swarm, form an organism, task shape matching, reconfiguration, planning and control.

A symbiotic approach is proposed in this study to address this different MRR challenges.This model will be used as a strategy to address and determine if the modular part is harmful or beneficial to the robotic system. The term symbiosis comes from the Greek word meaning living together. In biology a symbiosis refers to two or more organism living with each other. The partners in a symbiotic relationship are called symbionts and are dependent to each other. A symbiont may benefit, suffer, or may not be affected from the relationship A symbiosis is a relationship between species that can be beneficial, harmful and no effect. Symbiosis can be classified into different types such as mutualism, commensalism, and parasitism. Mutualism describe a relationship in which the organisms involve derive benefit; commensalism is where only one species benefit and the other member has no apparent effect or neutral; and parasitism is a relationship in which one organism benefit at the cost to the other member harm[6]. Organisms develop symbiotic relationship as a strategy to adapt and survive in an unpredictable ecosystem. A symbiotic multi robot organism help address the challenges 
in a collective roboticswhichcan result in extended reliability, adaptability and long-term independence of artificial system. In addition to new technology, this may lead to deeper understanding the phenomena of collective intelligence and artificial evolution[7].

\section{METHODOLOGY}

\subsection{Symbiotic Mathematical Model}

Mathematical model of symbiosis was established in [8]Refer to (1). This model shows the interaction between species thru the influence of their carrying capacity to each other. The model entities can pertain to biological, ecological, social and financial societies but in our case, it will be applied in a modular mobile robot as means of control, fault detection or diagnose depending on how the carrying capacity is defined.

$$
K i=A i+B i S i[N 1, N 2\}
$$

Symbiosis corresponds to the mutual interaction of species on the carrying capacity of each other. Carrying capacity is considered to be a function of the quantities Ni. The first term $A i$ is the carrying capacity of the given surrounding livelihood. The second term characterizes the carrying capacity produced by other species. The symbiotic coefficient $B i$ defines the intensity of producingor destroyingthecarrying capacity in the symbiotic relations. When $B i$ is positive it is considered as the production coefficient, when $B i$ is negative as the destruction coefficient. As seen below

$$
A i>0, B i \in(-\infty, \infty)
$$

Mutual interaction is characterized by the sign of the symbioticcoefficient $\mathrm{Bi}$, the symbiosis function

$$
\operatorname{Si}(\{N 1, N 2, \ldots\}) \geq 0
$$

The symbiotic functions depend on the number of species. Assuming that the effective carrying capacity is a linear combination of the natural carrying capacity, provided by nature, two symbiotic species. When the carrying capacity of an $i$-species is influenced by the mutual interactions with a $j$ species, the effective carrying capacity is represented as

$$
K i=A i+B i N i N j
$$

And if the carrying capacity of the i-species is influenced by the $j$-species without direct

interactions, as it happens in the case of commensalism, then the effective carrying capacity is given by the form

$$
K i=A i+B i N j
$$

The natural carrying capacity Ai is expected to be nonzero, which means that the speciescould exist without their symbionts. There can be situations when one symbiont is dependent with one another, so that one of the species cannot survive without the other and would correspond to zero carrying capacity. Main categories of symbiotic relations can be distinguished (mutualism, parasitism, and commensalism), depending on whether the influence of one species on another is positive, negative, or neutral.

Mutualism implies the relations in which both species extract some benefit from theirrelationship. Parasitism means that one will benefit, while the other is harm. Commensalism is a relation in which one of the species benefits, while the other is unaffected. The summary of relations is shown in the following inequalities below.

$B 1>0, B 2>0($ mutualism $)$

$B 1>0, B 2<0, B 1<0, B 2>0, B 1<0, B 2$

$<0$ (Parasitism)

$B 1>0, B 2=0, B 1=0, B 2>0($ Commensalism $)$

In addition to this classification, it is possible to distinguish different kinds of mutual interactions embodied in the form of the symbiosis function $\mathrm{Si}(\{\mathrm{Ni}\})$ obeying inequality before specifying this function $\mathrm{Si}(\{\mathrm{Ni}\})$, Symbiotic functions areassumed to be as ananalytical function and can be expanded in power series over the species populations [8].

In order for the symbiotic algorithm to be applied on the modular system. We need to identify the Carrying Capacity of each module. Carrying capacity can be identified as the certain task or mission that the whole system needed to accomplish. The carrying capacity can be the maximum velocity attain, the farthest distance to travel, the quickesttravel time, the ability to push, pull or transport certain objects, the ability traverse and climb certain terrain, total battery consumption or a combination of this different task. Symbiotic Function depends to the interaction of different modules and the number of populations.

\subsection{Modular Robot Design Model}

The base model that was used is a six-wheel independent drive with 3 modules. They are considered to have better performance, efficient power utilization and stability over other wheeled vehicles such as $4 \mathrm{wd}$. Due to advantages over conventional axle drive systemsall wheel independent drive vehicle is getting popularity in special purpose as well as commercial purpose vehicle. This configuration is good in obstacle climbing, off-road maneuvering, failure handling of few wheels, because of independent wheel motion control, used in exploration, search and rescue [9]. 
There are various research that uses a six wheel with 3 module body for climbing, transport and exploration [2][10][11]. The concept of [2] is to develop a detachable modular robot capable of cooperative climbing and multi agent exploration and will improve the task and exploration coverage butthere are a variety of optimal control problems that might pose in this system. [11] Develop an end-toendsystem for addressing task with modular robot and demonstrate that it can accomplish challenging multi part task. They use a SMORES-EP Modular robot that have 6 different configurations depending on the number of connected modules and the stair climber configuration has a total of 4 modules. [12] Uses an S-bot in a chain of three or more to transport the object. With these different studies they use 3 modules to accomplish certain task and a sixwheel configuration is ideal in an exploration and rover application. There have been lot of interest in optimizing formulation and control of actuators for six-wheel independent drive vehicle to maximize its exploration capabilities [9].

This application of the symbiotic approach in the MRR might aid the system in exploration and mission performance that are semi-autonomously or autonomously.A three module with six-wheel differential drive is chosenand will be model in a MATLAB Simulink to simulate a configuration carrying capacity. Inorder for the system to identify whether the module will do harm, benefit, or no effect to the robotic system.

This study will use a differential drive module per body. Equation (6) state that variable $\mathrm{x}$ can be described by the robot's location xR and $\mathrm{yR}$, forward velocity $v$, heading $\theta$, and angular velocity $\omega$. Mathematically, this is described as $\mathrm{x}=[\mathrm{xR} ; \mathrm{yR} ; v ; \theta ; \omega] \mathrm{T}$.

Taking our inputs as $u_{l}$ and $u_{r}$, the forces exerted by the left and right wheels respectively, system

$$
x=f(x)=\left[\begin{array}{c}
v \cos \theta \\
v \sin \theta \\
\frac{u l+u_{r}}{M}-b v \\
\omega \\
\frac{B\left(u_{l}-u_{r}\right)}{I z}-b_{r} \omega
\end{array}\right]
$$

where $\mathrm{M}$ is the vehicle mass, $\mathrm{b}$ is the linear drag constant, $\mathrm{B}$ is the length from the center ofthe vehicle to the wheel, Iz is the moment of inertia, and br is the turning drag constant. We assume the magnitude of each of input $\mathrm{u}_{\mathrm{rl}}$ is bounded by $\mathrm{u}_{\max }$ and the velocity is bounded by $\mathrm{v}_{\max }$. The proponent will use a differential ground drive robot for each module and will be simulated in a six wheeled configuration.

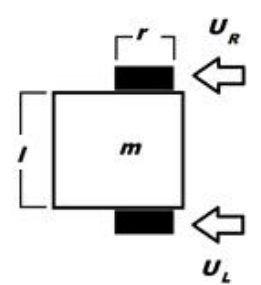

Figure 1:Two-wheel differential drive module

A single module body will be model as a differential drive robot. $\mathrm{L}$ as a wheelbase body and $\mathrm{r}$ as the wheel radius. The robot will be control by moving the two wheels. The input on the wheel on the left and the wheel on the right. As the configuration changes from $4 \mathrm{wd}$ to $6 \mathrm{wd}$ they will be simulated and check the response of the configuration pertaining to the action of each modules. If they will increase the carrying capacity of the whole system translating into velocity or distance. The configuration set-up which is the $4 \mathrm{wd}$ or $6 \mathrm{wd}$ will help determine if the modules are beneficial, harmful or no effect to the carrying capacity of the configuration. As mention in [2][10][11] they have certain advantage and disadvantages when they are in a collective manner or as an individual. They will be simulated by checking the response of the system when the whole module has inputs on their wheel and varying each modules wheel input to check the interaction of the modules in the simulated configuration.

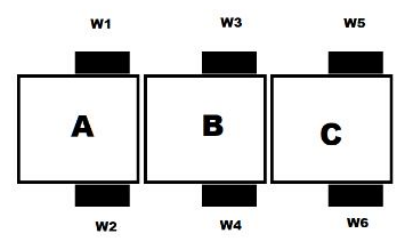

Figure 2: Propose six-wheel 3 module setup

Contact modelling was used in the modular body to approximate the physical phenomena of the forces acting on the body as well as the rolling of the tires. This help in the analysis of the mechanical system to identify the carrying capacity of the propose design mechanical system

A sphere to plane contact force library was used to simulate the design model of the modular body and compare the result of the individual and whole configuration. This contact force library implements a contact force between two bodies which is the sphere (wheels) and the plane(floor). To check the movement of the wheel with respect to different forces such as gravity and friction forces. Static and Kinematic Friction are set up in this library as well as the 
Arvin H. Fernando et al., International Journal of Emerging Trends in Engineering Research, 9(5), May 2021, $602-607$

density of the body to signify the effect of the gravitational phenomena.

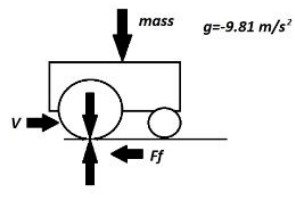

Figure 3: Contact Forces acting on a body

\section{RESULTS AND DISCUSSION}

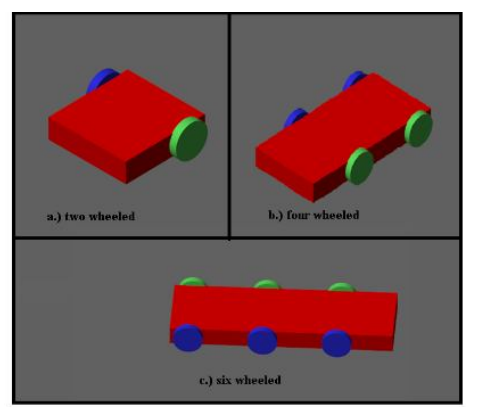

Figure 4:MATLAB model Simulink Design Model

The propose symbiotic mathematical was tested in a 3module body 6 wheel and simulated in MATLAB Simulink pertaining to the movement of wheels. A total of three configuration was tested in $2 \mathrm{wd}$. $4 \mathrm{wd}$ and $6 \mathrm{wd}$ set-up and then tested on one module moving the body. Figure 3 shows the design model of the module in the MATLAB Simulink . It was model in the MATLAB Simulink with estimated parameters of one module will have $\mathrm{L}=80 \mathrm{~mm}$ and $\mathrm{R}=$ $16 \mathrm{~mm}$ with thickness of $7 \mathrm{~mm}$ as the module size is reference to a micro mouse platform and other modular reconfiguring robots are at the micro size level. Contact forces are set to default such as gravity, kinetic and static friction. The density of the material is set to $1000 \mathrm{~kg} / \mathrm{m}^{3}$ to simulate the mass of the module. A ramp up signal was introducingto both wheelsand it yield a simulated velocity of $0.1596 \mathrm{~m} / \mathrm{s}$ in a one module differential drive set-up. It was tested on 10second time trial and the measure the distance that it recorded on the Simulinkscope is $1.589 \mathrm{~m}$ that can be seen in figure 4 . Dimension such as body and wheel size will affect the output result due to weight. In this set up we investigate effect of the module configuration to thecarrying capacity of the system which we define as velocity and distance. Different parameters and different set up will yield different result.

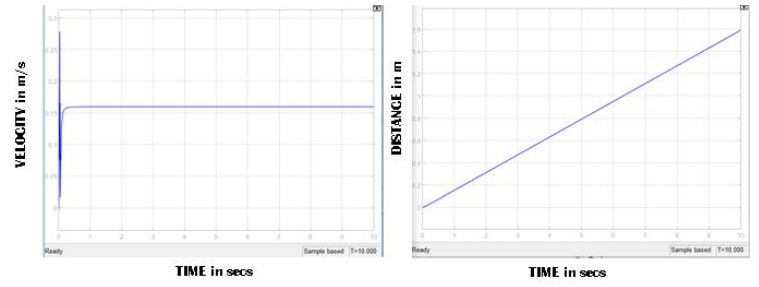

Figure 5:One module Velocity and Distance Result

Carrying Capacity may also vary and change because we are investigatingon a flat terrain checking if the distance and velocity changes. Configuration also have advantages and disadvantages as mention in [2][9][10][11]. Velocity and Distance capacity was tested for the $2 \mathrm{wd}$ one module body, 4 wd two module body and a 6 wd three module body. Every module will have the same carrying capacity as individual

however, with different configuration this will change. The simulated output results of the $4 \mathrm{wd}$ and $6 \mathrm{wd}$ can be seen in figure 6 and 7. The 4 wd two module set up resulted into an increase of carrying capacity in terms of velocity and distance. There is a slight increase from 0.1596 to $0.16 \mathrm{~m} / \mathrm{s}$ as simulated while distance travel increases from 1.589 to $1.595 \mathrm{~m}$. The initial start-up as seen in the graph is faster compare to the single module configuration. However, result from the 3-module configuration resulted to a decrease in velocity and distance due to the total weight having a velocity of 0.1594 and distance travel of $1.57 \mathrm{~m}$ in 10 seconds. The initial start-up of velocity was also slow in comparison to the two-module set up even though it is in 6wd configuration This result give a decrease of carrying capacity in terms of velocity and distance travel however if we use the stability, terrain capabilities and other 6wd properties as the carrying capacity the result will change.

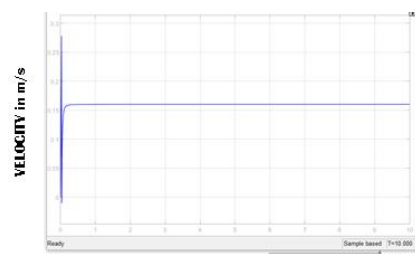

TIME in secs

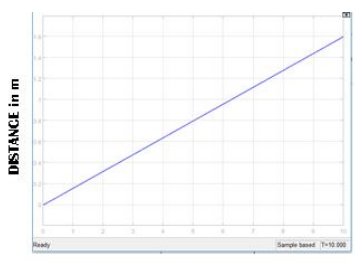

TIME in secs
Figure 6:Two Module Velocity and Distance Result

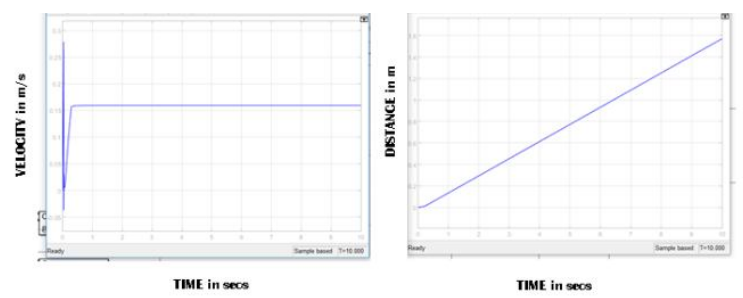

Figure 7: Three Module Velocity and Distance Result 
Using (1) and the symbiotic functions of the carrying capacity of an individual module we calculated the symbiotic coefficient.The two modules configuration gives a positive symbiotic coefficient of 0.0157 and the threemodule configuration gives off a negative coefficient of0.04919 using the velocity and distance as a carrying capacity.However, the result is almost negligible due to the degree of scale and limitation of the definition of carrying capacity. Further investigation and study are needed for the overall carrying capacity. Other simulation was also tested by not giving input on the other wheels and letting them freely rotate with respect to the applied force of module and the result differs. Figure 9-10 shows the result that The system was not able to travel or startup if one module is only functioning at three module configurations due to the weight.

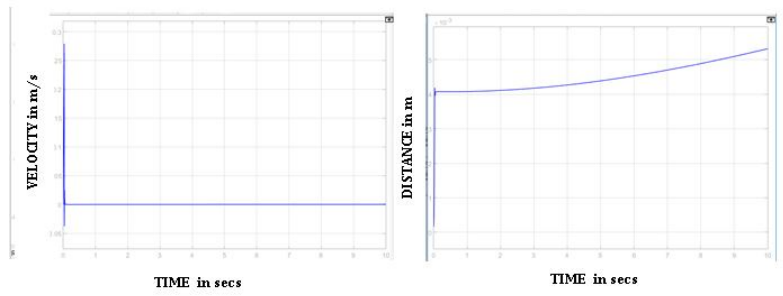

Figure 8: Three Module Velocity and Distance front moduleonly

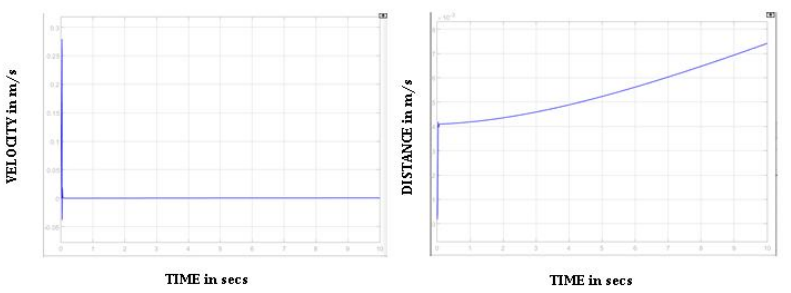

Figure 9: Three Module Velocity and Distance middle module only
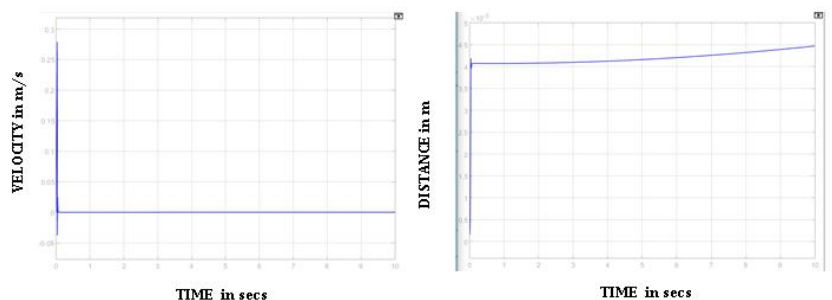

Figure 10: Three Module Velocity and Distance back module only

In Table 1 and 2 shows the result of the 2 and 3 module configurations set up. The $\mathrm{M}$ variable represents the module body M1,M2 and M3, respectively. It can be seen that the weight of the module directly affects the velocity and distance. Having only the rear module drive whether it is a 2 or 3 configuration shows that it has a difficulty in moving. It might be due to the initial start-up torque due to the rolling resistance in the simulation the result in actual testing might change. In a three-module configuration it shows that having the front and mid functioning shows an increase in result. Running two modules and letting one module freely rotate was also tested in the simulation. Having the two modules carrying the body and the result that there is not much significant changes having two modules running on the front have a faster and longer distance effect.

Table 1: Summary of two module velocity and distance simulation result

\begin{tabular}{|c|c|c|c|}
\hline M1 & M2 & $\begin{array}{c}\text { Velocity } \\
(\mathbf{m} / \mathbf{s})\end{array}$ & $\begin{array}{c}\text { Distance } \\
(\mathbf{m})\end{array}$ \\
\hline on & on & 0.16 & 1.595 \\
\hline on & off & 0.1597 & 1.591 \\
\hline off & on & 0.000137 & 0.004788 \\
\hline
\end{tabular}

Table 2:. Summary of three module velocity and distance simulation result

\begin{tabular}{|c|c|c|c|c|}
\hline M1 & M2 & M3 & $\begin{array}{c}\text { Velocity } \\
(\mathrm{m} / \mathrm{s})\end{array}$ & $\begin{array}{c}\text { Distance } \\
(\mathrm{m})\end{array}$ \\
\hline on & on & on & 0.1594 & 1.57 \\
\hline on & on & off & 0.1598 & 1.592 \\
\hline on & off & off & 0.0002296 & 0.005317 \\
\hline on & off & on & 0.1594 & 1.57 \\
\hline off & on & on & 0.1596 & 1.588 \\
\hline off & off & on & 0.00008195 & 0.00447 \\
\hline off & on & off & 0.007413 & 0.00486 \\
\hline
\end{tabular}

As stated in [8] Carrying capacity will vary depending in the number of species which can be seen in the result of simulation. It was also found out that the carrying capacity will also depend on the role and function of the species. The effect of weight shows the slight decrease in speed however if we take consideration the battery consumption and configuration capability the carrying capacity will change. We clearly needed to define how we will identify or set the overall carrying capacity if it is totality or certain function that is why in a very critical situation sometimes semiautonomous are much prefer compare to the fully autonomous where experience and judgement matter on the end result.

\section{CONCLUSION AND RECOMMENDATION}

In this paper, A concept of symbiotic approach is proposed to a modular mobile robot to address the hardware and software challenges. Analyzing the Symbiotic Coefficient per module is required and necessary to identify if it will do harm, benefit, or no effect to the carrying capacity of the 
robotic configuration system. A base model of a six-wheel 3 module body differential drive was simulated and investigatedthe application of the symbiotic mathematical model that will response to the capacity of certain task. The MATLAB simulation is used as a tool to check the proposed strategy. Results shows that the response of the modular body varies depending on what wheel is functioning whether it is givingwheel speed and freewheeling.

The performance of the body change whether the front, mid or rear module is functioning. This might help in maximizing the velocity and distance efficiency of a six wheel or a long module vehicle body such as trains or any future modular transport system.

Further simulation and validation of the study is needed. It was also seen in the result of the simulation that the role and function of the module or species affects the carrying capacity. Other parameters should also be consideredwhether the species or module could be harmful, beneficial to the system. This study might contribute to a new development of strategy in modular mobile robot application in the near future.

\section{REFERENCES}

1. AL. Christensen Fault Detection in Autonomous Robot, Université Libre de Bruxelles, 2008

2. SH Turlapati, A Srivastava, KM Krishna, SV Shah Detachable modular robot capable of cooperative climbing and multi agent exploration 2017 IEEE International Conference on Robotics and Automation (ICRA), 255-260, 2017

3. J Seo, J Paik, M Yim Modular reconfigurable robotics Annual Review of Control, Robotics, and Autonomous Systems 2, 63-88,2019

4. A Brunete, A Ranganath, S Segovia, JP de Frutos, $M$ Hernando, Current trends in reconfigurable modular robot's design International Journal of Advanced Robotic Systems 14 (3), 1729881417710457, 2017

5. Yim M, Shen WM, Salemi B, Rus D, Moll M. Modular self-reconfigurable robot

systems IEEE Robotics \& Automation Magazine 2007

6. H. Aydt, S. Turner, W. Cai, M. LowSymbiotic Simulation Systems: An Extended Definition Motivated by Symbiosis in Biology $22^{\text {nd }}$ PADS Roma, Italy, 2008

7. P. Levi, S. Kernbach, Symbiotic Multi-Robot Organisms: Reliability, Adaptability, Evolution. Springer, 2010

8. VI Yukalov, EP Yukalova, D Sornette Modeling symbiosis by interactions through species carrying capacities Nonlinear Phenomena 241 (15), 1270-1289,2012

9. R. Prasad, Y. MaHierarchical Control Coordination Strategy of Six Wheeled Independent Drive(6WID) Skid Steering Vehicle IFAC 2019

10. SH Turlapati, M Shah, SP Teja, A Siravuru, SV Shah, Stair climbing using a compliant modular robotIEEE/RSJ 2015

11. G Jing, T Tosun, M Yim, H Kress-Gazit An Endto-End System for Accomplishing Tasks with Modular Robots, Robotics: Science and Systems 2016

12. R Groß, E Tuci, M Dorigo, M Bonani, F Mondada Object transport by modular robots that selfassemble Proceedings IEEE International Conference on Robotics and Automation 2006

13. GK Fourlas, S Karkanis, GC Karras, KJ Kyriakopoulos Model based actuator fault diagnosis for a mobile robot IEEE International Conference on Industrial Technology (ICIT), 7984,2014

14. AA Bandala, EP Dadios, RRP Vicerra, LAG Lim Swarming algorithm for unmanned aerial vehicle (uav) quadrotors-swarm behavior for aggregation, foraging, formation, and tracking JACII 2014

15. AA Bandala, EP Dadios 2012 Development and design of mobile robot with IP-based vision systemTENCON IEEE R10 2012

16. JRB Del Rosario, JG Sanidad, AM Lim, PSL Uy, AJC Bacar, MAD Cai, Modelling and Characterization of a Maze-Solving Mobile Robot Using Wall Follower Algorithm Applied Mechanics and Materials 446, 1245-1249,2014

17. A Fernando, L GanLim Velocity analysis of a six wheel modular mobile robot using MATLABSimulink, IOP Conference Series: Materials Science and Engineering 1109 (1), 012037,2021

18. Steve Miller Simscape Multibody ContactForcesLibrary (https://www.mathworks.c om/matlabcentral/fileexchange/47417-simscapemultibody-contact-forces-library), MATLAB Central File Exchange 2020

19. Gavin Nielsen Micro Mouse Modelling Simscape MultiBody, 2017 